\title{
Improving insulin resistance with Antrodia cinnamomea mycelium powder to induce a hypoglycemic effect in dexamethasone-induced insulin-resistant rats
}

\author{
YUAN-CHIANG CHUNG ${ }^{1,2}$, CHUNG-YUH TZENG ${ }^{2,3}$, YING-I CHEN $^{2}$, SHU-WEI CHANG $^{2}$, TAI-HAO HSU ${ }^{4}$, \\ WAI-JANE $\mathrm{HO}^{2}$, YUEH-HSIUNG KUO ${ }^{5,6}$, PEI-HSIU $\mathrm{HUNG}^{7}$ and SHIH-LIANG CHANG ${ }^{2,8,9}$
}

${ }^{1}$ Department of Surgery Chung-Kang Branch, Cheng-Ching Hospital, Taichung 40764; ${ }^{2}$ Department of Medicinal Botanicals and Health Applications, Da-Yeh University, Changhua 51591; ${ }^{3}$ Department of Orthopedics, Taichung Veterans General Hospital, Taichung 40705; ${ }^{4}$ Department of Bioindustry Technology, Da-Yeh University, Changhua 51591; ${ }^{5}$ Department of

Chinese Pharmaceutical Sciences and Chinese Medicine Resources, China Medical University; ${ }^{6}$ Department of Biotechnology, Asia University, Taichung 41354; ${ }^{7}$ Division of Traditional Chinese, Ditmanson Medical Foundation Chia-Yi Christian Hospital, Chiayi 60002; ${ }^{8}$ School of Chinese Medicine, China Medical University, Taichung 40402; ${ }^{9}$ College of Biotechnology and Bioresource, Da-Yeh University, Changhua 51591, Taiwan, R.O.C.

Received June 15, 2017; Accepted September 21, 2017

DOI: $10.3892 / \mathrm{mmr} .2017 .8259$

\begin{abstract}
Insulin resistance is a major factor in type II diabetes development, occurring when insulin levels are normal, but do not have normal interactions with adipose, muscle or liver tissue. The present study aimed to explore the hypoglycemic effect of Antrodia cinnamomea (AC) mycelium powder by evaluating its impact on insulin resistance and plasma free fatty acid (FFA) levels in steroid-induced insulin-resistant (SIIR) rats. Male Wistar rats were administered dexamethasone for 5 days to induce insulin resistance. The SIIR rats were subsequently randomly assigned into three experimental groups (EGs) and a control group (CG), where saline was orally administered. The EGs were orally administered different doses of $\mathrm{AC}(100,200$ or $500 \mathrm{mg} / \mathrm{kg})$ and an optimal dose for further study was determined. Changes in plasma insulin and glucose levels were calculated to investigate the hypoglycemic effect of AC. To evaluate insulin resistance, the homeostasis
\end{abstract}

Correspondence to: Professor Shih-Liang Chang, Department of Medicinal Botanicals and Health Applications, Da-Yeh University, 168 University Road, Dacun, Changhua 51591, Taiwan, R.O.C.

E-mail: slchang@mail.dyu.edu.tw

Abbreviations: AC, Antrodia cinnamomea; CG, control group; $\mathrm{DM}$, diabetes mellitus; EG, experimental group; FFA, free fatty acid; GLUT-4, glucose transporter 4; HOMA-IR, homeostasis model assessment-estimated insulin resistance; IRS-1, insulin receptor substrate-1; PI3K, phosphoinositide 3-kinase; SIIR, steroid-induced insulin resistant; TZD, thiazolidinedione

Key words: Antrodia cinnamomea, dexamethasone, free fatty acid, insulin signal transduction proteins, insulin resistance model assessment-estimated insulin resistance of the SIIR rats was determined. Changes in plasma FFA levels were detected and levels of insulin signal proteins (IRS-1, GLUT-4 and PI3K) were analyzed by western blot to elucidate AC's mechanism of action. The SIIR rats exhibited significantly decreased plasma glucose levels in the first $30 \mathrm{~min}$, with plasma FFA levels displaying a marked downward trend $(\mathrm{P}<0.05)$ when they were administered the optimal dose of AC (200 mg/kg). The decrease in plasma glucose and FFA levels was significantly larger in the EG compared to the $\mathrm{CG}$, and insulin signal protein levels were also significantly increased $(\mathrm{P}<0.05)$. The hypoglycemic effect observed may be due to decreased plasma FFA levels and increased expression of intracellular insulin signal proteins. Furthermore, insulin sensitivity was enhanced, indicating that $\mathrm{AC}$ acts as an insulin sensitizer in insulin resistant animal models.

\section{Introduction}

Diabetes mellitus (DM) is one of the top 10 most prevalent diseases worldwide, ranking fourth in Taiwan. Individuals with DM are at an increased risk of developing eye disease, foot lesions, nerve degeneration, cerebrovascular disease, cardiovascular disease, hypertension and renal disease (1). The pathology of type II DM results from insulin resistance and/or a lack of insulin secretion, which ultimately increases plasma glucose levels. This hyperglycemic state stimulates insulin secretion and induces hyperinsulinemia (2). The clinical symptoms of insulin resistance include dyslipidemia, hypertension, glucose intolerance, hyperuricemia or gout, central obesity, impaired blood coagulation or hyper-coagulation, hyperandrogenism like symptoms, fatty liver and coronary vascular disease (3). These chronic complications not only threaten the life of those diagnosed with type II DM, but also puts a heavy burden on medical resources and the social economy (4). 
Todate, the best methods for improving insulin resistance are exercise (5), smoking cessation (6) and medications including metformin, a biguanide (7) and thiazolidinediones (TZDs), a class of oral hypoglycemic agents (8). In complementary and alternative medicine, it has also been demonstrated that electroacupuncture on the Zhongwan (CV12) acupoint or the bilateral Zusanli (ST36) acupoints may lower plasma glucose and enhance insulin sensitivity (9-11). Among the aforementioned methods, the use of medication is the most effective and convenient for the management of type II DM, but can result in adverse side effects. Therefore, it is important to identify other methods with fewer side effects that can improve insulin resistance.

Antrodia cinnamomea (AC) is a Taiwanese fungus species that grows in the hollow trunk of the Cinnamomum kanehirai tree. Previous investigations have indicated that AC may suppress tumor formation (12), enhance the immune system (13), inhibit viral activity and protect the liver (14). It is often used in Taiwanese folk medicine. Some evidence has also indicated that $\mathrm{AC}$ can act as an anti-oxidant, improve hypertension and decrease plasma lipids (15-17). Ergostatrien-3 $\beta$-ol (EK100) from the $\mathrm{AC}$ was evaluated for its hypoglycemic effects and was demonstrated to improve diabetes and dyslipidemia in mice fed a high-fat diet. EK100 treatment also resulted in decreased visceral adipocyte size and reduced the ballooning degeneration of hepatocytes. Levels of glucose transporter 4 (GLUT-4) protein and Akt phosphorylation in skeletal muscle are also significantly increased in EK100 treated mice (18). In addition, antroquinonol extracted from the mycelium of AC effectively inhibited dipeptidyl peptidase-4 activity and AMP-activated protein kinase (AMPK) activation (19). Dehydroeburicoic acid from AC prevented the development of diabetic and dyslipidemic states in streptozotocin-induced diabetic mice, through the regulation of GLUT-4, peroxisome proliferator activated receptor $\alpha$, fatty acid synthase and AMPK phosphorylation (20). An extract made from the fruiting body of AC lowered liver triglyceride and total cholesterol levels (17) and enhanced the production of superoxide dismutase, catalase and glutathione peroxidase (21).

In addition to the aforementioned effects of $\mathrm{AC}$, its therapeutic effects on insulin resistance have also been investigated (22-24), but the mechanism by which AC enhances insulin sensitivity has not been completely elucidated, particularly for steroid-induced insulin-resistance (SIIR) $(25,26)$. Steroids are widely prescribed and are known to cause insulin resistance. Patients with DM treated with steroids commonly require an increased dose of insulin. Thus, the aim of the present study was to use the SIIR rat as a model to explore the hypoglycemic and insulin resistance improving effects of orally administered $\mathrm{AC}$ and to investigate the mechanisms underlying its hypoglycemic and insulin resistance improving properties.

\section{Materials and methods}

Preparation of AC mycelium. AC mycelium was cultured on solid-state cereal medium (provided by the Chair Professor Wai-Jane Ho, Da-Yeh University, Changhua, Taiwan) for $85-90$ days at $21-23^{\circ} \mathrm{C}$ in the dark. The solid medium was mainly barley-supplemented with yeast extract and glucose. At the end of the culturing period, cultures were harvested, dried and ground into powder for subsequent experiments. The final yield from the solid-state culture was $\sim 20 \%$ of the original dry weight of cereal medium (Fig. 1A). The powdered culture was then mixed with normal saline to produce oral solutions at the concentrations of 100, 200 and $500 \mathrm{mg} / \mathrm{kg}$ body weight (BW). The solutions were subsequently stored in a $4^{\circ} \mathrm{C}$ refrigerator ready for use.

High-performance liquid chromatography (HPLC) assay. Components of the AC powder methanol extract were assessed by HPLC assay with a configured module using a Waters Alliance 2695 with auto-sample injection (Waters XTerra column, 4.6x250 mm, $5 \mu \mathrm{m}$; mobile phase A, water; mobile phase B, acetonitrile; injection volume, $10 \mu \mathrm{l}$; detector, PDA; detector wavelength, qualitative $250 \mathrm{~nm}$, quantitative $280 \mathrm{~nm}$; temperature, $25^{\circ} \mathrm{C}$; flow rate, $1.0 \mathrm{ml} / \mathrm{min}$; Waters Corporation, Milford, MA, USA). The peaks of the retention time from the analysis of the AC methanol extract were compared with the peaks of the internal standard dissolved in methanol solution (provided by Professor Yueh-Hsiung Kuo, China Medical University, Taichung, Taiwan).

Animal models. A total of 24 Male Wistar rats aged 8-10 weeks, 250-300 g BW, were obtained from the BioLASCO (Taipei, Taiwan). They were housed in rooms at $25 \pm 1^{\circ} \mathrm{C}$ with relative humidity of $65 \pm 5 \%$. The rats were acclimatized in an alternating $12 \mathrm{~h}$ light/12 h dark cycle with free access to water and standard rat chow. After 1 week of adaptation, the rats were randomly assigned into experimental and control groups. The Institutional Animal Care and Use Committee of Da-Yeh University approved the methods of this study according to the national guidelines for the Care and Use of Animals.

A previously published research protocol was followed to establish the SIIR rat model (26). At 8 weeks, rats were intraperitoneally (i.p.) injected with dexamethasone at a dose of $1 \mathrm{mg} / \mathrm{kg}$ daily for 5 days. It was concluded that insulin resistance was successfully induced when their measured fasting plasma glucose levels were higher than $150 \mathrm{mg} / \mathrm{dl}$. Tests were performed at 8:00 a.m. and all SIIR rats were fasted before each test and anesthetized with pentobarbital (40 mg/kg i.p.) (11).

Experimental protocols. The SIIR rats were randomly divided into four groups and administered the following treatments once: Group A, control group $(\mathrm{n}=6), 1 \mathrm{ml} / \mathrm{kg}$ oral saline; group B (n=6), $100 \mathrm{mg} / \mathrm{kg} \mathrm{AC}$; group C (n=6), $200 \mathrm{mg} / \mathrm{kg}$; and group D ( $\mathrm{n}=6), 500 \mathrm{mg} / \mathrm{kg} \mathrm{AC}$. The three experimental groups (EGs) were force-fed the AC solution. Every AC sample was shock homogenized prior to each feeding. The plasma glucose levels were assayed with a commercial enzymatic method using Glucose HK stable liquid reagent (Randox Laboratories Ltd., Crumlin, UK). The percentage hypoglycemic activity was calculated as follows: [plasma glucose levels at $30 \mathrm{~min}$ (or $60 \mathrm{~min}$ ) - plasma glucose levels at $0 \mathrm{~min} /$ plasma glucose levels at $30 \mathrm{~min}$ (or $60 \mathrm{~min}$ )] x 100. The optimal dose group for inducing hypoglycemia was determined and used in subsequent experimental procedures.

Assay of plasma free fatty acid (FFA) levels. Plasma FFA levels were measured using a non-esterified fatty acid kit 

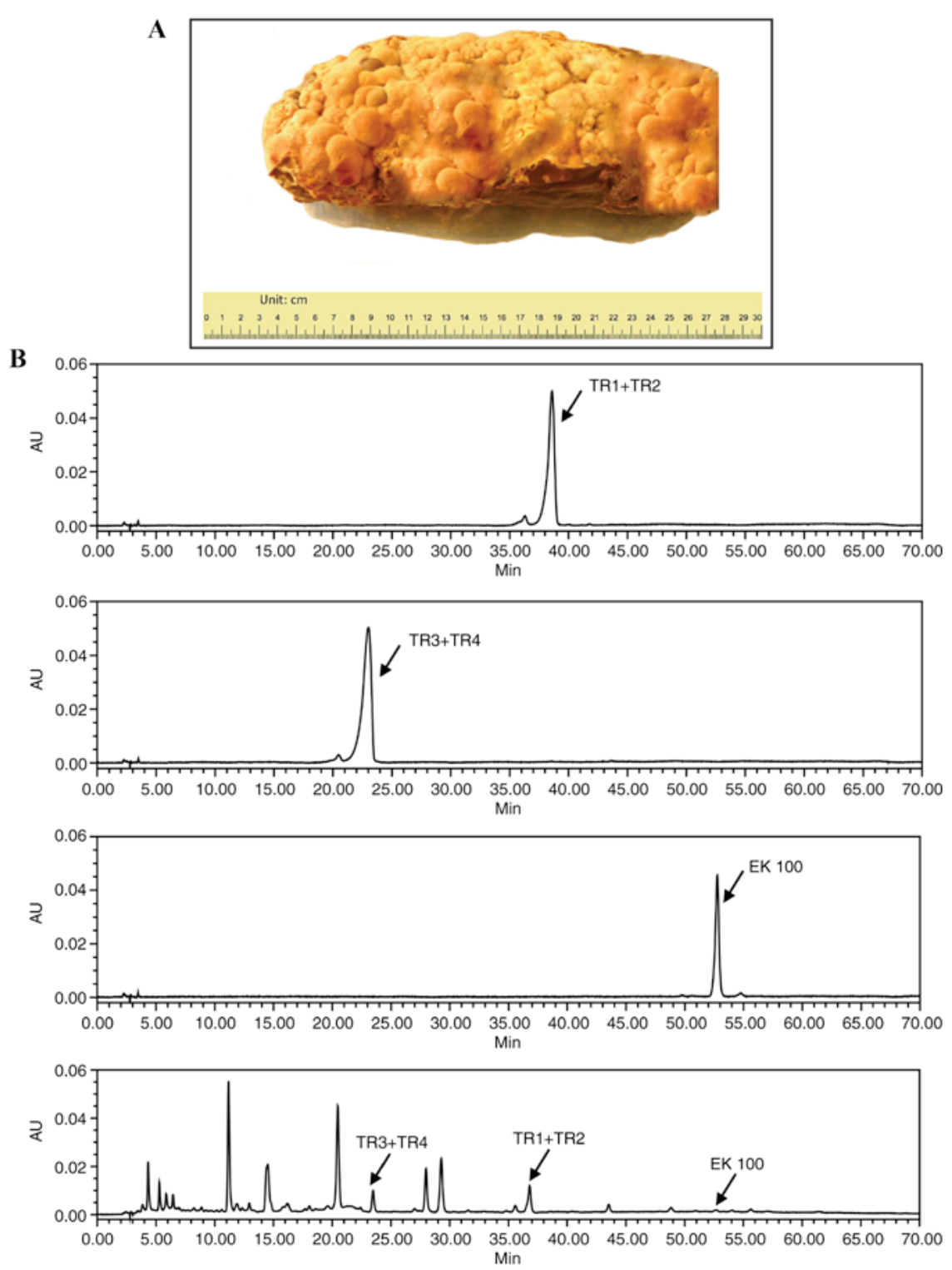

Figure 1. (A) Sample of AC from solid-state cereal cultures. (B) HPLC analysis of AC mycelium powder methanol extract. HPLC, high-performance liquid chromatography; AC, Antrodia cinnamomea; TR1, eburicoic acid; TR2, dehydroeburicoic acid; TR3, sulphurenic acid; TR4, dehydrosulphurenic acid; EK100, ergostatrien-3ß-ol.

(Randox Laboratories Ltd.). FFA levels were measured via an ELISA. As described in our previous study (25), plasma FFA was transformed into a purple adduct for subsequent detection by automatic spectrophotometer (COBAS MIRA Plus system; Roche Diagnostics, Basel, Switzerland).

ELISA of plasma insulin levels and resistance test. A Mercodia ultrasensitive rat insulin ELISA kit (cat. no. 10-1251-01; Mercodia AB, Uppsala, Sweden) was used to detect plasma insulin levels. The homeostatic model assessment of insulin resistance (HOMA-IR) was calculated using the following formula: [fasting plasma insulin levels $(\mu \mathrm{U} / \mathrm{ml}) \mathrm{x}$ fasting plasma glucose $(\mathrm{mmol} / \mathrm{l})] / 22.5(27,28)$.

Western blot analysis. The samples were minced coarsely and homogenized by an ultrasonic processor (VCX 750; Sonics and Materials Inc., Newtown, CT, USA) in a radioimmunoprecipitation assay lysis buffer with the protease inhibitor, phenylmethylsulfonyl fluoride (Santa Cruz Biotechnology, Inc., Dallas, TX, USA). The muscle extracts were centrifuged at $16,440 \mathrm{x} g$ at $4^{\circ} \mathrm{C}$ for $1 \mathrm{~h}$, and the supernatants were measured using a spectrophotometer. A total of $90 \mu \mathrm{g} / \mathrm{ml}$ protein was separated by $8 \%$ SDS-PAGE, and the proteins were transferred to a polyvinylidene fluoride (PVDF) membrane for western blotting. The PVDF membrane was then submerged in 5\% non-fat milk to block the nonspecific binding sites in the membrane at $25^{\circ} \mathrm{C}$ for $1 \mathrm{~h}$. The membrane was incubated overnight with anti-insulin receptor substrate-1 (IRS-1; 1:200, sc-559), anti-GLUT-4 (1:200, sc-7938) and anti-phosphoinositide 3-kinase (PI3K; 1:200, sc-376112) antibodies (all from Santa Cruz Biotechnology, Inc.) at $4{ }^{\circ} \mathrm{C}$ in the refrigerator. Finally, the membranes were incubated with goat anti-rabbit immunoglobulin G-horseradish peroxidase antibodies (GTX213110-01, 1:2,000; GeneTex, Inc., Irvine, CA, USA) for at $25^{\circ} \mathrm{C}$ for $1 \mathrm{~h}$, and specific bands were detected by an enhanced chemiluminescence kit (Clarity $^{\mathrm{TM}}$ 
and Clarity Max ${ }^{\mathrm{TM}}$ Western ECL Blotting Substrates; Bio-Rad Laboratories, Inc., Hercules, CA, USA) The bands were quantified using optical densitometry (Gel-Pro analysis version 4.0; Media Cybernetics, Rockville, MD, USA). The actin bands were used as an internal loading control, the results are presented as a ratio of signal-to-actin $(11,26)$.

Statistical analysis. The experimental results are presented as the mean \pm standard error in each group $(n=6)$. The statistical analysis of the results was performed using the Student's t-test or one-way analysis of variance, with a least significant difference post hoc test. $\mathrm{P}<0.05$ was considered to indicate a statistically significant difference.

\section{Results}

HPLC assay. HPLC analysis detected eburicoic acid + dehydroeburicoic acid (TR1+TR2), sulphurenic acid + dehydrosulphurenic acid (TR3+TR4) and EK100 (standard retention times: 36-38, 20-23, and $52.8 \mathrm{~min}$, respectively). The AC sample contained TR1+TR2, TR3+TR4, and EK100 (Fig. 1B).

Hypoglycemic effect of AC in SIIR. The SIIR rats in the EGs were administered different doses of AC (100, 200 or $500 \mathrm{mg} / \mathrm{kg}$ ) and the $\mathrm{CG}$ rats were administered normal saline. At $30 \mathrm{~min}$ after treatment, the plasma glucose levels in the rats administered 200 and $500 \mathrm{mg} / \mathrm{kg}$ of $\mathrm{AC}$ was significantly lower than those in the $\mathrm{CG}(\mathrm{P}<0.05)$. After $60 \mathrm{~min}$, the plasma glucose levels in all EGs was significantly lower than those in the $\mathrm{CG}(\mathrm{P}<0.01$; Fig. 2A). At $30 \mathrm{~min}$ after treatment administration, the hypoglycemic activity in the 200 and $500 \mathrm{mg} / \mathrm{kg}$ groups was 21.69 and $15.22 \%$ respectively, which was significantly greater than that of the CG $(3.33 \%, \mathrm{P}<0.05)$. After $60 \mathrm{~min}$, the hypoglycemic activity of the 200 and $500 \mathrm{mg} / \mathrm{kg}$ groups was 26.59 and $26.86 \%$, respectively, both of which were significantly greater than that of the CG $(9.74 \%, \mathrm{P}<0.05$; Fig. 2B). No specific adverse events, including no mortality, normal activity, water and food intake were observed following each experiment at any dose.

Hypoglycemic effect of the optimal dose of $A C$. The above results indicated that $200 \mathrm{mg} / \mathrm{kg}$ AC was optimal to achieve a hypoglycemic effect. Therefore, this particular dose was used in the subsequent experiments. Thirty minutes after administration of $200 \mathrm{mg} / \mathrm{kg}$ of $\mathrm{AC}$, the plasma glucose level decreased from $155.33 \pm 23.12$ to $125.81 \pm 29.81 \mathrm{mg} / \mathrm{dl}(\mathrm{P}<0.05$ vs. baseline), and to $113.69 \pm 14.15 \mathrm{mg} / \mathrm{dl}$ after $60 \mathrm{~min}(\mathrm{P}<0.05$ vs. baseline).

Plasma insulin and HOMA-IR levels in SIIR rats following administration of $200 \mathrm{mg} / \mathrm{kg}$ AC. Following oral administration of $200 \mathrm{mg} / \mathrm{kg} \mathrm{AC}$, plasma insulin levels were detected in serum samples taken from the femoral vein using an ELISA kit. Plasma insulin levels increased from $0.35 \pm 0.41$ to $0.51 \pm 0.30 \mathrm{pmol} / 1$ at 30 min after $\mathrm{AC}$ administration, with a significant elevating trend compared to those of the $\mathrm{CG}$, in which plasma insulin levels changed from $0.11 \pm 0.07$ to $0.07 \pm 0.02 \mathrm{pmol} / 1$ after $30 \mathrm{~min}$. There was a significant difference between the levels of insulin in the EG and the $\mathrm{CG}$ at $30 \mathrm{~min}$ (Fig. 3A).
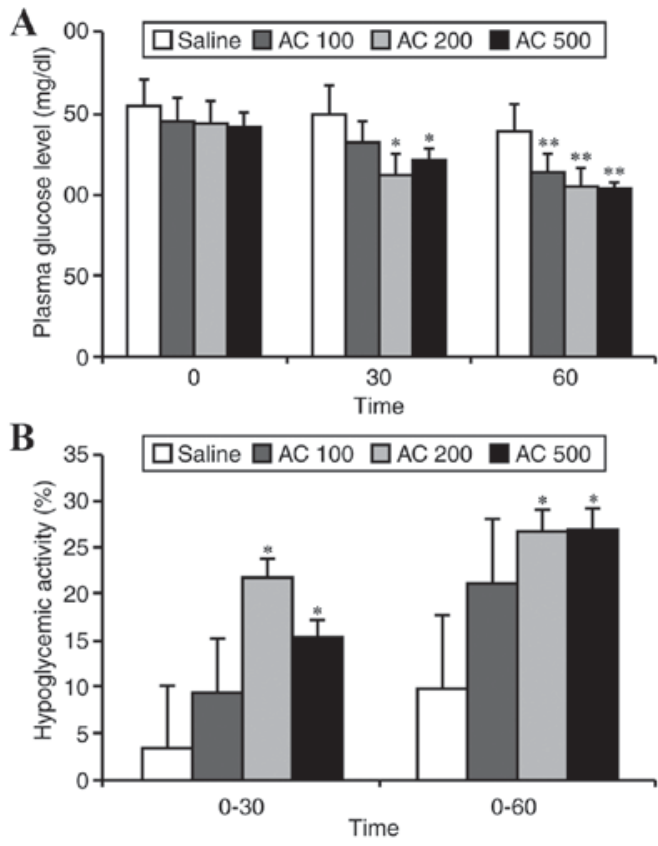

Figure 2. (A) Plasma glucose levels after administration of different AC doses to SIIR rats. (B) The hypoglycemic activity of different doses of AC in the SIIR Wistar rats $\left({ }^{*} \mathrm{P}<0.05,{ }^{* *} \mathrm{P}<0.001\right.$ vs. saline). AC, Antrodia cinnamomea; SIIR, steroid-induced insulin-resistant; AC 100, $100 \mathrm{mg} / \mathrm{kg} ;$ AC 200, $200 \mathrm{mg} / \mathrm{kg}$; AC 500, $500 \mathrm{mg} / \mathrm{kg}$.
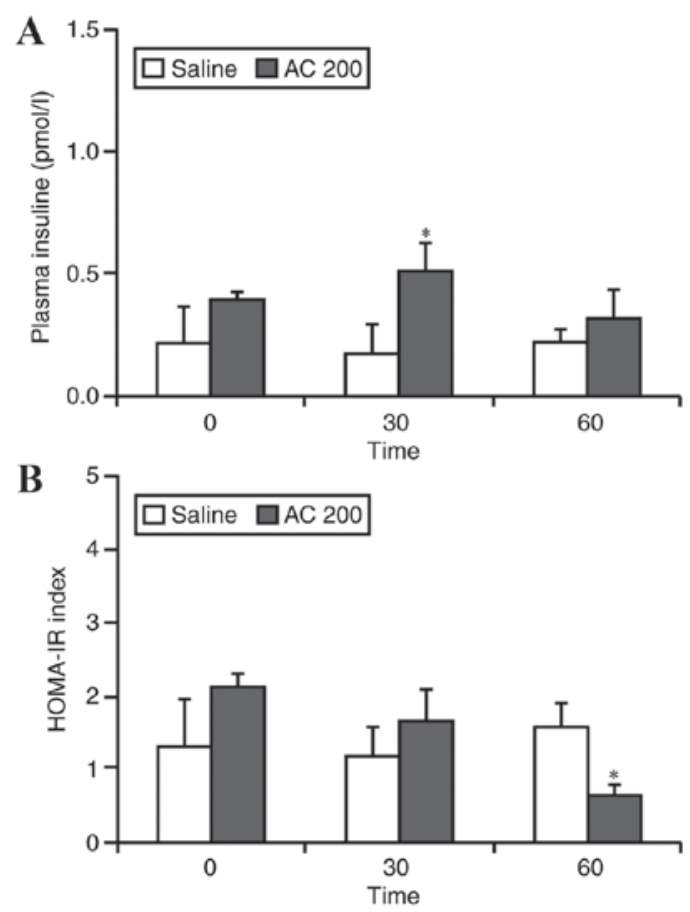

Figure 3. (A) Plasma insulin levels after administration of $200 \mathrm{mg} / \mathrm{kg}$ AC 200 to the SIIR rats. (B) The HOMA-IR index after administration of $200 \mathrm{mg} / \mathrm{kg}$ AC to the SIIR rats. ${ }^{*} \mathrm{P}<0.05$ vs. saline. AC, Antrodia cinnamomea; AC 200 $200 \mathrm{mg} / \mathrm{kg}$; SIIR, steroid-induced insulin-resistant; HOMA-IR, homeostasis model assessment-estimated insulin resistance.

There were no significant differences in HOMA-IR levels between the EG and the $\mathrm{CG}$ at baseline and $30 \mathrm{~min}$ after oral administration of $200 \mathrm{mg} / \mathrm{kg}$ of AC. However, HOMA-IR levels in the EG were significantly decreased compared 


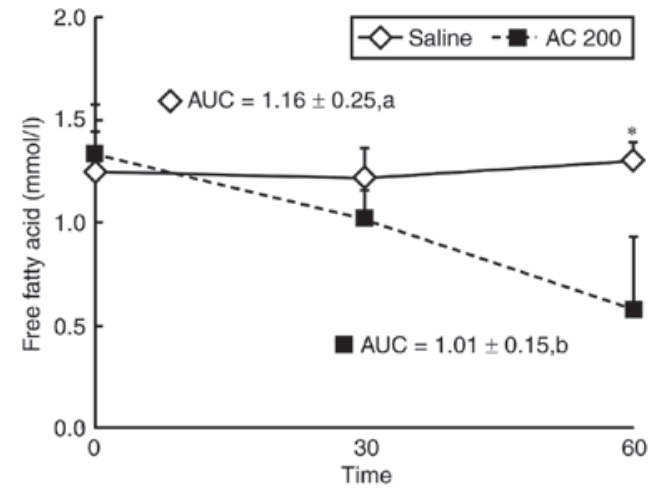

Figure 4. AUC of the plasma free fatty acid concentration curve; a $>$ b ("P $<0.05$ vs. AC 200). AC, Antrodia cinnamomea; AC 200, $200 \mathrm{mg} / \mathrm{kg}$; AUC, area under the curve.

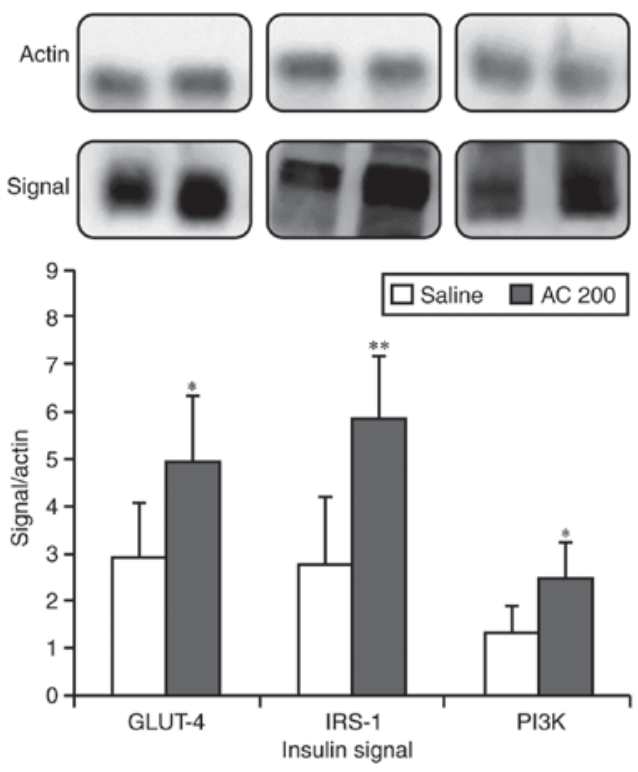

Figure 5. Alterations in signaling proteins for IRS-1, GLUT-4 and PI3K in the AC administered SIIR rats. ${ }^{*} \mathrm{P}<0.05,{ }^{* *} \mathrm{P}<0.01$ vs. saline. IRS-1, insulin-receptor substrate-1; GLUT-4, glucose transporter-4; PI3K, phosphoinositide 3-kinase; SIIR, steroid-induced insulin-resistant; AC, Antrodia cinnamomea; AC 200, $200 \mathrm{mg} / \mathrm{kg}$.

with the $\mathrm{CG}$ at $60 \mathrm{~min}$ after $\mathrm{AC}$ administration $(1.53 \pm 0.22$ vs. $0.83 \pm 0.50$ respectively, $\mathrm{P}<0.05$; Fig. $3 \mathrm{~B}$ ).

Plasma FFA levels in SIIR rats following $200 \mathrm{mg} / \mathrm{kg} \mathrm{AC}$. At 60 min, plasma FFA levels in the EG had decreased from $1.33 \pm 0.24$ to $0.58 \pm 0.35 \mathrm{mmol} / 1$, and were significantly decreased $(15.69 \pm 3.97 \%)$ compared with in the CG, in which plasma FFA levels were increased from $1.25 \pm 0.19$ to $1.30 \pm 0.09 \mathrm{mmol} / 1(5.5 \pm 0.80 \%)$. Additionally, the area under the curve of plasma FFA in the EG $(1.01 \pm 0.15)$ was significantly smaller than that of the CG $(1.16 \pm 0.25, \mathrm{P}<0.05$; Fig. 4).

Expression of insulin signal proteins following AC administration. The levels of insulin signal proteins, IRS-1, GLUT-4, and PI3K, were significantly elevated compared to the CG following $\mathrm{AC}$ administration. The fold changes (EG/CG) were 1.71, 2.10 and 1.87, respectively (Fig. 5).

\section{Discussion}

Metabolic syndrome has gained growing attention in recent years, particularly in developed countries. Overeating and an aging society contributes to the increasing incidence of metabolic syndrome, and insulin resistance may be a major factor that contributes to the development of type II DM (29). Therefore, preventing metabolic syndrome or correcting insulin resistance may help to slow the development of type II DM. There are many oral hypoglycemic agents and insulin sensitizers like TZDs available, but the side effects of these drugs limit their use (30). An agent with fewer side effects that can manage of plasma glucose levels is desirable for patients, particularly one that can also improve insulin resistance.

$\mathrm{AC}$ is a valuable medicinal fungus found in the forests of Taiwan. It grows naturally in the hollow trunk of the old Cinnamomum kanehirai tree or through artificial culture on host wood sections. Due to its versatile biomedical activities, AC is highly valued (12-16) and Taiwanese forests are threatened with the illegal logging of Cinnamomum kanehirai trees. This study developed a solid-state culture method for $\mathrm{AC}$ product to combat this serious illegal logging problem. Additionally, a standard operating procedure was developed for the AC solid-state culture method, to verify the quality of each batch (provided by Chair Professor Wai-Jane Ho from Da-Yeh University; Fig. 1A). The possible toxicity or potential side effects of the AC mycelium power may be relative to the dose, but in the present study, $100 \%$ of the rats survived following administration of the highest dose $(500 \mathrm{mg} / \mathrm{kg})$. Physiological signs including respiration, hair color and food and water intake, were all normal after 1 week of AC administration. As metabolic syndrome is a chronic condition requiring long-term treatment, the potential toxic effects of $\mathrm{AC}$ are very important and should be investigated. However, acute and subacute toxicity tests were not the aim of the present study.

The AC powder methanol extract was analyzed using HPLC to verify the presence of active hypoglycemic components, EK100, TR1+TR2 and TR3+TR4 (Fig. 1B). These components were previously reported to be bioactive and present in $\mathrm{AC}(18,20,31)$. There is concern that the solid-state culture method may result in variation of the components between batches and different strengths of bioactivity, but this HPLC analysis method allows for the quality control of each batch. Prior to the study, experimental doses were determined in preliminary tests by administering various doses to a SIIR rat once a week to establish a small, medium, and high dose $(100,200$, and $500 \mathrm{mg} / \mathrm{kg})$ appropriate for rats. Thus, the doses used are different than what would be appropriate in a clinical situation.

The hypoglycemic effect of AC was subsequently evaluated and supported by the findings of the present study. The optimal oral dose to achieve a hypoglycemic effect was determined to be $200 \mathrm{mg} / \mathrm{kg} \mathrm{AC}$, and this was used to explore the mechanism of action of AC.

Previous research has confirmed the hypolipidemic effect of $\mathrm{AC}(17,20,31,32)$, supporting the results of the present study that indicated a decrease in plasma FFA levels. To establish the SIIR animal model, steroids were administered, causing an increase in plasma FFA levels and the development of insulin 
resistance $(11,25)$. AC improved insulin resistance by lowering plasma FFA levels in the SIIR state. Steroids are frequently used to treat inflammatory diseases and the impairment of insulin sensitivity is a problematic side effect, particularly in patients with type II DM. The results of this study demonstrate that AC may improve insulin resistance caused by the steroid administration.

The increase in plasma insulin in the EG was not significant at the $60 \mathrm{~min}$ time-point compared with the $\mathrm{CG}$, which might have been due to the duration of plasma insulin secretion within this studied animal model due to oral administration of the AC powder. However, HOMA-IR at the 60 min time-point indicated an improvement in insulin resistance following $\mathrm{AC}$ treatment; enhanced insulin secretion induced by AC was recently reported in MIN6 cells (33). Therefore, this AC may elevate plasma insulin and improve insulin resistance.

With respect to signal transduction proteins, AC caused an increase in IRS-1, PI3K and GLUT-4. Previous studies have indicated that AC stimulates AMPK to enhance GLUT-4 translocation and activates the peroxisome proliferator activated receptor $\alpha(\operatorname{PPAR} \alpha)$ to decrease plasma FFA levels, which may complement the insulin signaling pathway to result in a hypoglycemic effect and improvement in insulin resistance $(18,20,32,34)$. In our previous studies, we also used the SIIR animal model to test the hypoglycemic effect of the Xylaria nigripes (Xn) fungus and the Gardenia jasminoides (GJ) plant $(26,35)$. However, Xn was found to exert a serotonin-associated hypoglycemic effect, and PPAR activation was key to the hypoglycemic effect of GJ. This differs from the mechanism of AC identified in this study, in which a decrease in plasma FFA levels was observed.

Administration of $200 \mathrm{mg} / \mathrm{kg}$ AC to SIIR rats resulted in a decrease in plasma glucose levels, which was closely associated with a decrease in plasma FFA levels. Furthermore, an increase in the expression of insulin signaling proteins (GLUT-4, IRS-1 and PI3K) was observed with improved insulin resistance. These results indicate that $\mathrm{AC}$ acts as an insulin sensitizer in insulin resistant animals. Due to the use of an animal model in this study, results cannot be applied to a clinical situation. Thus, a randomized controlled trial of AC mycelium powder should be performed to determine the clinical dosage and enhance its effect on insulin resistance.

\section{Acknowledgements}

This study was supported by grants provided by the Ministry of Science and Technology (grant no. 104-2632-E-212-001), Taichung Veterans General Hospital and Da-Yeh University joint project (grant no. TCVGH-DYU-1058304) and Cheng Ching Hospital and Da-Yeh University Joint Project (grant no. CCGH-DYU-106-001 \& CCGH-DYU-104-001) in Taiwan. This study was also supported by a grant from China Medical University under the Aim for Top University Plan of the Ministry of Education, Taiwan (grant no. CHM106-5-2) and Taiwan Ministry of Health and Welfare Clinical Trial Center, Taiwan (grant no. MOHW106-TDU-B-212-113004) for the analysis of HPLC.

\section{References}

1. Annadurai T, Vasanthakumar A, Geraldine P and Thomas PA: Variations in erythrocyte antioxidant levels and lipid peroxidation status and in serum lipid profile parameters in relation to blood haemoglobin A1c values in individuals with type 2 diabetes mellitus. Diabetes Res Clin Pract 105: 58-69, 2014.

2. Colussi G, Catena C, Lapenna R, Nadalini E, Chiuch A and Sechi LA: Insulin resistance and hyperinsulinemia are related to plasma aldosterone levels in hypertensive patients. Diabetes Care 30: 2349-2354, 2007.

3. DeFronzo RA and Ferrannini E: Insulin resistance. A multifaceted syndrome responsible for NIDDM, obesity, hypertension, dyslipidemia, and atherosclerotic cardiovascular disease. Diabetes Care 14: 173-194, 1991.

4. Lamounier-Zepter V, Ehrhart-Bornstein M and Bornstein SR: Insulin resistance in hypertension and cardiovascular disease. Best Pract Res Clin Endocrinol Metab 20: 355-367, 2006.

5. Hecksteden A, Grütters T and Meyer T: Associations between acute and chronic effects of exercise on indicators of metabolic health: A pilot training trial. PLoS One 8: e81181, 2013.

6. Eliasson B, Attvall S, Taskinen MR and Smith U: Smoking cessation improves insulin sensitivity in healthy middle-aged men. Eur J Clin Invest 27: 450-456, 1997.

7. Inzucchi SE, Maggs DG, Spollett GR, Page SL, Rife FS, Walton V and Shulman GI: Efficacy and metabolic effects of metformin and troglitazone in type II diabetes mellitus. N Engl J Med 338: 867-872, 1998.

8. Jermendy G and Csermely P: Thiazolidinediones-a new class of oral antidiabetic drugs. Orv Hetil 142: 1547-1554, 2001 (In Hungarian).

9. Chang SL, Lin JG, Chi TC, Liu IM and Cheng JT: An insulin-dependent hypoglycaemia induced by electroacupuncture at the Zhongwan (CV12) acupoint in diabetic rats. Diabetologia 42: 250-255, 1999.

10. Chang SL, Lin KJ, Lin RT, Hung PH, Lin JG and Cheng JT: Enhanced insulin sensitivity using electroacupuncture on bilateral Zusanli acupoints (ST 36) in rats. Life Scie 79: 967-971, 2006.

11. Tzeng CY, Lee YC, Chung JJ, Tsai JC, Chen YI, Hsu TH, Lin JG, Lee KR and Chang SL: $15 \mathrm{~Hz}$ electroacupuncture at ST36 improves insulin sensitivity and reduces free fatty acid levels in rats with chronic dexamethasone-induced insulin resistance. Acupunct Med 34: 296-301, 2016.

12. Li SL, Huang ZN, Hsieh HH, Yu WC, Tzeng WY, Lee GY, Chen YP, Chang CY and Chuu JJ: The augmented anti-tumor effects of Antrodia camphorata co-fermented with Chinese medicinal herb in human hepatoma cells. Am J Chin Med 37: 771-783, 2009.

13. Song AR, Qin D, Zhao C, Sun XL, Huang F, Kong C and Yang S: Immunomodulatory effect of polysaccharides extracted from the medicinal mushroom Antrodia camphorata (higher Basidiomycetes) in specific pathogen-free chickens. Int J Med Mushrooms 16: 95-103, 2014.

14. Yue PY, Wong YY, Wong KY, Tsoi YK and Leung KS: Current evidence for the hepatoprotective activities of the medicinal mushroom Antrodia cinnamomea. Chin Med 8: 21, 2013.

15. Hsiao G, Shen MY, Lin KH, Lan MH, Wu LY, Chou DS, Lin CH, Su CH and Sheu JR: Antioxidative and hepatoprotective effects of Antrodia camphorata extract. J Agric Food Chem 51: 3302-3308, 2003

16. Yue PY, Wong YY, Chan TY, Law CK, Tsoi YK and Leung KS: Review of biological and pharmacological activities of the endemic Taiwanese bitter medicinal mushroom, Antrodia camphorata (M. Zang et C. H. Su) Sh. H. Wu et al. (higher Basidiomycetes). Int J Med Mushrooms 14: 241-256, 2012.

17. Lai MN, Ko HJ and Ng LT: Hypolipidemic effects of antrodia cinnamomea extracts in high-fat diet-fed hamsters. J Food Biochem 36: 233-239, 2012.

18. Kuo YH, Lin $\mathrm{CH}$ and Shih CC: Ergostatrien-3 $\beta$-ol from Antrodia camphorata inhibits diabetes and hyperlipidemia in high-fat-diet treated mice via regulation of hepatic related genes, glucose transporter 4, and AMP-activated protein kinase phosphorylation. J Agric Food Chem 63: 2479-2489, 2015.

19. Weng CF, Chen CP, Shivaji SR and Hsu CY: Compounds from antrodia camphorate and their use in treatment of diabetes mellitus. US Patent 20150203430 A1. Filed January 22, 2014; issued July 23, 2015. 
20. Kuo YH, Lin $\mathrm{CH}$ and Shih CC: Antidiabetic and antihyperlipidemic properties of a triterpenoid compound, dehydroeburicoic acid, from antrodia camphorata in vitro and in streptozotocin-induced mice. J Agric Food Chem 63: 10140-10151, 2015.

21. Wen CL, Chang CC, Huang SS, Kuo CL, Hsu SL, Deng JS and Huang GJ: Anti-inflammatory effects of methanol extract of Antrodia cinnamomea mycelia both in vitro and in vivo. J Ethnopharmacol 137: 575-584, 2011

22. Kuo MT, Tseng WP, Tseng TL and Kuo YY: Method for promoting insulin secretion by using compounds and extracts isolated from antrodia camphorata. US Patent 20160038549 A1. Filed August 7, 2015; issued February 11, 2016.

23. Tsai PJ, Wang C, Chou CJ and Huang WC: Method for controlling obesity using Antrodia camphorata. US Patent 20150157673 A1. Filed December 5, 2013; issued June 11, 2015.

24. Park TS: Composition for prevention or treatment of obesity, dyslipidemia, fatty liver or insulin resistance syndrome comprising camphene as active ingredients. US Patent 20120035274 A1. Filed March 18, 2009; issued February 9, 2012.

25. Lin RT, Tzeng CY, Lee YC, Ho WJ, Cheng JT, Lin JG and Chang SL: Acute effect of electroacupuncture at the Zusanli acupoints on decreasing insulin resistance as shown by lowering plasma free fatty acid levels in steroid-background male rats. BMC Complement Altern Med 9: 26, 2009.

26. Chen YI, Cheng YW, Tzeng CY, Lee YC, Chang YN, Lee SC, Tsai CC, Chen JC, Tzen JT and Chang SL: Peroxisome proliferator-activated receptor activating hypoglycemic effect of Gardenia jasminoides Ellis aqueous extract and improvement of insulin sensitivity in steroid induced insulin resistant rats. BMC Complement Altern Med 14: 30, 2014.

27. Takai T and Sakura H: Insulinogenic index, HOMA-beta, disposition index. Nihon Rinsho 70 (Suppl 3): S459-S464, 2012 (In Japanese).
28. Haffner SM, Miettinen H and Stern MP: The homeostasis model in the San Antonio Heart Study. Diabetes Care 20: 1087-1092, 1997.

29. Chillarón JJ, Flores-Le-Roux JA, Goday A, Benaiges D, Carrera MJ, Puig J, Cano-Pérez JF and Pedro-Botet J: Metabolic syndrome and type-1 diabetes mellitus: Prevalence and associated factors. Rev Esp Cardiol 63: 423-429, 2010 (In Spanish).

30. Rizos CV, Elisaf MS, Mikhailidis DP and Liberopoulos EN: How safe is the use of thiazolidinediones in clinical practice? Expert Opin Drug Saf 8: 15-32, 2009.

31. Peng $\mathrm{CH}$, Yang MY, Yang YS, Yu CC and Wang CJ: Antrodia cinnamomea prevents obesity, dyslipidemia, and the derived fatty liver via regulating AMPK and SREBP signaling. Am J Chin Med 45: 67-83, 2017.

32. Kuo YH, Lin CH, Shih CC and Yang CS: Antcin K, a triterpenoid compound from Antrodia camphorata, displays antidiabetic and antihyperlipidemic effects via glucose transporter 4 and AMP-activated protein kinase phosphorylation in muscles. Evid Based Complement Alternat Med 2016: 4867092, 2016.

33. Vong CT, Tseng HH, Kwan YW, Lee SM and Hoi MP: Antrodia camphorata increases insulin secretion and protects from apoptosis in MIN6 Cells. Front Pharmacol 7: 67, 2016.

34. Kuo YH, Lin CH and Shih CC: Dehydroeburicoic acid from Antrodia camphorata prevents the diabetic and dyslipidemic state via modulation of glucose transporter 4, peroxisome proliferator-activated receptor alpha expression and AMP-activated protein kinase phosphorylation in high-fat-fed mice. Int J Mol Sci 17: E872, 2016

35. Chen YI, Tzeng CY, Cheng YW, Hsu TH, Ho WJ, Liang ZC, Hsieh CW, Tzen JT and Chang SL: The involvement of serotonin in the hypoglycemic effects produced by administration of the aqueous extract of Xylaria nigripes with steroid-induced insulin-resistant rats. Phytother Res 29: 770-776, 2015. 